\title{
Controllable band gap engineering of new polymer solar cell materials
}

The Institute of Polymer Optoelectronic Materials \& Devices, South China University of Technology, Guangzhou, in close collaboration with the Department of Electrical and Electronic Engineering, The University of Hong Kong, has shown that the band gap and energy levels of conjugated polymers can be finely tuned using a two-dimensional-like donor- $\pi$-acceptor (D- $\pi$-A) molecular design, which is of important for polymer solar cell applications. The study is reported in Volume 54 of Science China Chemistry because of its significant research value.

Bulk-heterojunction (BHJ) polymer solar cells (PSCs) have attracted considerable attention because of unique advantages over traditional silicon-based solar cells such as low cost, light weight, and the potential to produce flexible, large area devices by roll-to-roll manufacturing. In most cases, BHJ-type solar cells use a phase-separated blend of organic electron donor and acceptor components, where a conjugated polymer and fullerene derivative are often used as the donor and acceptor, respectively. To achieve high performance BHJ-type PSCs, the electron-donating conjugated polymer needs to strongly absorb a broad range of solar light, and possess good hole mobility and a highest occupied molecular orbital (HOMO) level that maximize the short-circuit current $\left(J_{\mathrm{sc}}\right)$ and open-circuit voltage $\left(V_{\mathrm{oc}}\right)$, respectively. This requires simultaneous control of the band gap, energy levels and molecular packing of the conjugated polymer.

To realize ready, precise control of the electronic and optical properties of conjugated polymers, Prof. Huang and collaborators proposed a new two-dimensional-like D- $\pi$-A conjugated polymer design strategy. The main chains of the polymers were constructed of electron-rich donor moieties (such as fluorene, silafluorene, carbazole and triphenylamine), while the electron-withdrawing acceptor groups were located at the ends of the side chains and connected to the electron-donating moieties on the main chains via $\pi$-bridges. This produces polymers with a two-dimensional-like structure with an electron-rich conjugated main chain and D- $\pi$-A conjugated side chains. The band gaps and absorption spectra of the resulting polymers can be effectively tuned by controlling the push-pull interactions of the side chains simply by incorporating different acceptor groups, while a relatively deep HOMO energy level is maintained by control over moieties in the main chain (J Am Chem Soc, 2009, 131: 13886; Macromolecules, 2010, 43: 5262; Chem Mater, 2010, 22: 6444).

As an important step forward in their research on two-dimensional-like D- $\pi$-A conjugated polymers, Dr. Duan and Prof. Huang used three kinds of acceptors (malononitrile, 1,3-diethyl-2-thiobarbituric acid and 2-(1,2-dihydro-1-oxoinden-3-ylidene) malononitrile) with different electron-withdrawing abilities as pendant groups in D- $\pi$-A polymers. They showed that the band gaps of the polymers could be successfully tuned from 2.05 to $1.61 \mathrm{eV}$, while their relatively deep HOMO energy levels of $\sim-5.35 \mathrm{eV}$ were preserved. Through collaboration with the Department of Electrical and Electronic Engineering, The University of Hong Kong, the authors demonstrated that these two-dimensional-like conjugated polymers exhibit promising photovoltaic properties, with a high $V_{\text {oc }}$ of over $0.9 \mathrm{~V}$ achieved for all polymers despite their different band gaps (Sci China Chem, 2011, 54: 685-694).

"It is quite interesting that the band gaps and energy levels of the polymers were effectively tuned by simply attaching different acceptor groups, while their relatively deep HOMO energy levels of $\sim-5.35 \mathrm{eV}$ were preserved." said one journal reviewer. "The results are interesting to the organic solar cell community" said another.

This work was supported by the National Natural Science Foundation of China (50990065, 5101003, 51073058 and 20904011), the National Key Basic Research Program of China (2009CB623601), the UGC grant (400897) of The University of Hong Kong and Hong Kong Research Grants Council (HKU\#712108 and HKU\#712010).

See the articles: Duan C H, Wang C D, Liu S J, et al. Two-dimensional like conjugated copolymers for high efficiency bulk-heterojunction solar cell application: Band gap and energy level engineering. Sci China Chem, 2011, 54: 685-694

Huang F, Chen K S, Yip H L, et al. Development of new conjugated polymers with donor- $\pi$-bridge-acceptor side chains for high performance solar cells. J Am Chem Soc, 2009, 131: 13886-13887

Duan C H, Cai W Z, Huang F, et al. Novel silafluorene-based conjugated polymers with pendant acceptor groups for high performance solar cells. Macromolecules, 2010, 43: 5262-5268

Duan C H, Chen K S, Huang F, et al. Synthesis, characterization, and photovoltaic properties of carbazole-based two-dimensional conjugated polymers with donor- $\pi$-bridge-acceptor side chains. Chem Mater, 2010, 22: 6444-6452

Open Access This article is distributed under the terms of the Creative Commons Attribution License which permits any use, distribution, and reproduction in any medium, provided the original author(s) and source are credited. 